\title{
Comparison of the tolerability and efficacy of intra-rectal lidocaine gel with peri-prostatic nerve block as anaesthetic techniques for prostate biopsy
}

Musliu Adetola Tolani* ${ }^{*}$, Muhammed Ahmed, Ahmad Tijjani Lawal, Lovely Fidelis, Abdullahi Sudi, Babatunde Kolapo Hamza, Mudi Awaisu, Oyelowo Nasir, Ahmad Bello and Hussaini Yusuf Maitama

\begin{abstract}
Background: Prostate biopsy is a painful procedure. However, over the years, varied opinions exist among urologists about the methods of achieving optimal pain relief for the procedure. The purpose of this study was to compare the tolerability of administration and the efficacy of intra-rectal lidocaine gel with peri-prostatic nerve block for this procedure.

Methods: From June 2016 to June 2017, 110 patients who met the study criteria were randomized to receive either intra-rectal lidocaine gel (Group 1) or an apical peri-prostatic nerve block (Group 2) for prostate biopsy. Their level of pain perception during the anaesthesia administration and the prostate biopsy was assessed using the Numeric Rating Scale. Other biopsy-related complications and patient satisfaction were also evaluated. Grouped data were compared using student $t$ test and Chi-square with $p<0.05$ considered significant.

Results: The pain score during the administration of anaesthesia was $1.6 \pm 1.9$ and $3.7 \pm 2.1$ for the intra-rectal lidocaine gel and peri-prostatic nerve block groups, respectively $(p=0.001)$. However, during the prostate biopsy, the pain score was $6.8 \pm 2.2$ and $2.9 \pm 1.9$ for the intra-rectal lidocaine gel and peri-prostatic nerve block groups, respectively $(p=0.001)$. There was a significantly lower rate of satisfaction ( $45.3 \%$ versus $86.8 \%, p=0.001)$ in the lidocaine gel group.
\end{abstract}

Conclusions: Although intra-rectal lidocaine gel administration is better tolerated by patients, a peri-prostatic nerve block is more effective and provides better patient satisfaction than intra-rectal lidocaine gel when used as anaesthesia for prostate biopsy.

Keywords: Prostate biopsy, Intra-rectal lidocaine gel, Peri-prostatic nerve block, Pain relief

\section{Background}

Prostate cancer is the second most common cancer among men globally [1]. Histology of a biopsy specimen obtained from the prostate is essential in its diagnosis. Methods of obtaining prostate biopsies have advanced

*Correspondence: adetolatolani@yahoo.com

Division of Urology, Department of Surgery, Ahmadu Bello University/ Ahmadu Bello University Teaching Hospital, P.M.B. 06, Shika-Zaria, Kaduna State, Nigeria over the years; from a limited lesion targeted biopsy to the systematic sampling of the whole gland and from digitally directed biopsy to trans-rectal ultrasound-guided biopsy [2, 3].

Patients experience varying degrees of pain during prostate biopsy. This occurs as a result of the introduction and movement of the ultrasound probe in the rectum, the injection of local anaesthetic as well as due to the penetration of the biopsy needle into the prostate capsule [4-7]. Studies have shown that varying 
levels of pain relief are felt by patients receiving local, regional or general anaesthesia for prostate biopsy $[5,6$, $8,9]$. However, there is a great recognition of the use of local anaesthesia for the procedure because of its ease of administration, cost and relatively fewer safety concerns compared to regional or systemic anaesthesia [7, 10].

The local anaesthetic approaches which have been used include the instillation of intra-rectal lidocaine gel and the peri-prostatic nerve block, which may be carried out through basolateral or apical techniques [7, 9, 11-13]. Although the use of intra-rectal lidocaine gel instillation seems quite attractive because of its wide availability and simplicity, there have been concerns about its efficacy in prostate biopsy as a result of its direct absorption into the haemorrhoidal circulation, rather than crossing through the rectal wall into and around the peri-prostatic nerves. On the other hand, trans-rectal ultrasound-guided periprostatic nerve block facilitates blockade of the capsular sensory fibres which are located around the prostate [13$15]$. It is presently regarded as the gold standard for pain relief during prostate biopsy.

This study aimed to compare the tolerability of administration and efficacy of intra-rectal lidocaine gel with peri-prostatic nerve block as techniques of anaesthesia for prostate biopsy.

\section{Methods}

\subsection{Study population and selection criteria}

All patients seen in our Urology Division from June 2016 to June 2017 with indications for prostate biopsy were considered for inclusion in the study. This included those with abnormal findings suggestive of prostate cancer on digital rectal examination or trans-rectal ultrasound prostate scan and those with elevated serum prostatespecific antigen $>4 \mathrm{ng} / \mathrm{ml}$ and prostate-specific antigen density $>0.15 \mathrm{ng} / \mathrm{ml} / \mathrm{g}$. Patients with allergy to lidocaine, acute urinary tract infection and acute painful conditions of the anorectal region like anal fissure or haemorrhoids were excluded. Other exclusion criteria include those with visual impairment, which is necessary for pain scoring and patients with loss of sensation in the lower part of the body.

This study was approved by the Institutional Health Research Ethics Committee and was carried out in accordance with the Declaration of Helsinki for experiments involving humans. Informed consent was obtained from all patients recruited for the study. A total of 110 consecutive patients were randomized into two equal groups, the intra-rectal lidocaine gel group (group 1) and the peri-prostatic nerve block group (group 2) by balloting from a sealed envelope.

\subsection{Study protocol}

The biodata, clinical details, prostate volume and prostate-specific antigen (PSA) data of the patients were obtained and recorded. Patients taking non-steroidal anti-inflammatory drugs were instructed to stop it 7 days before the biopsy, those on warfarin to stop it 3 days before the biopsy and those on analgesia to stop it the night before the procedure. Intravenous Gentamicin $(80 \mathrm{mg})$ was administered as antibiotic prophylaxis 30 min before the procedure.

The patients were consecutively placed on the left lateral decubitus position. For the patients in Group 1, $10 \mathrm{ml}$ of $2 \%$ intra-rectal lidocaine gel was instilled into the rectum. For those in Group 2, a $6.5 \mathrm{MHz}$ ultrasound probe was inserted into the rectum and oriented in the sagittal plane to locate the prostate apex. A size 22G, 7-inch spinal needle was then inserted under the guidance of an ultrasound probe with a needle guide facility and advanced to the prostate apex. Thereafter, the stylet was removed and the needle hub inspected for bleeding to exclude intravascular injection. Subsequently, peri-prostatic injection of $5 \mathrm{ml}$ of $2 \%$ plain lidocaine was administered first at $4^{\circ}$ clock position and then at the $8^{\circ}$ clock position of the prostate apex to achieve a bilateral apical block while ensuring that there was no resistance to the infiltration. Adequate infiltration was confirmed by the separation of tissue planes away from the ultrasound probe, after which the trans-rectal ultrasound probe was removed.

Systematic sextant biopsies were taken 5 min after the application of the anaesthesia. This was done under ultrasound guidance using a biopsy guide parallel to the ultrasound probe with size 18G TruCut biopsy needle inserted through the rectal wall into the prostate parenchyma in all patients. All ultrasound images were displayed on a Mindray Digital Ultrasonic Diagnostic Imaging System, DP-20 Model. The whole procedure was done by the same researcher using the specified protocol for each group.

Numeric Rating Scale was used for pain assessment. It is an 11-point scale having whole numbers integers $(0-10)$ listed along a horizontal line with "0" at one extreme representing "no pain" and "10" at the other extreme representing the worst pain felt [16]. Each patient was asked to select a whole number on the Numeric Rating Scale that best reflects the intensity of their pain, immediately after the administration of the local anaesthetic agent and also immediately after the prostate biopsy procedure with the help of a research assistant who was blinded on the technique of local anaesthesia used for the patient. This number was recorded on the proforma as the pain score by the research doctor. The patient's satisfaction with the prostate biopsy procedure was assessed using a 
dichotomous scale based on a "Yes" or "No" response format. The possibility of accepting a repeat biopsy by the patient, if necessary, was also assessed and recorded.

Patients were reviewed immediately after the procedure and followed up in the urology clinic, weekly for 2 weeks, after the procedure for possible complications.

\subsection{Statistical analysis}

The data were analysed using Statistical Package for Social Sciences (SPSS) software version 20. All continuous variables were summarized as mean \pm standard deviation for parametric data and median with range for nonparametric data. Categorical variables were summarized as frequencies with percentages. Independent samples $t$ test was used for the comparison of pain scores, while Mann-Whitney U test was used for comparison of prostate-specific antigen and prostate volume values. Chi-square test was used for the comparison of complications and satisfaction rates. The $p$-value was considered to be significant if $<0.05$.

\section{Results}

During the study period, two patients in each group were lost to follow up and excluded from the study. A total of 53 patients per group were finally analyzed. The age incidence of suspected prostate cancer patients biopsied is shown in Fig. 1. The overall mean age was $66.8 \pm 7.4$ years, while the median prostate-specific antigen and prostate volume were $18.6(4.0-103.2) \mathrm{ng} /$ $\mathrm{ml}$ and 55.2 (12.1-280.7) grams, respectively. The age, prostate-specific antigen and prostate volume were similar between the groups $(p>0.05)$ (Table 1$)$.

In comparison with the patients in the peri-prostatic nerve block group, those who received intra-rectal lidocaine gel had a significantly lower pain score during anaesthetic administration $(p=0.001)$. However, the reverse was the case during the prostate biopsy (Table 2). A large number of patients, 22 (41.5\%), had a pain score of 0 during intra-rectal lidocaine gel instillation, while many of them, $16(30.2 \%)$, had a pain score of 5 during peri-prostatic nerve block injection (Fig. 2). On the other hand, during the prostate biopsy, the most severe pain (pain score of 10) was recorded in the intrarectal lidocaine gel group in $12(22.6 \%)$ patients, while many of the patients, 14 (26.4\%), had a pain score of 2 in the peri-prostatic nerve block group (Fig. 3).

Seventy $(66 \%)$ of the patients were satisfied with the biopsy procedure, while 79 (74.5\%) would accept repeat biopsy. The rate of satisfaction and possible acceptance of repeat biopsy was significantly lower in the lidocaine gel group ( $p=0.001$ and $p=0.002$, respectively) (Table 2).

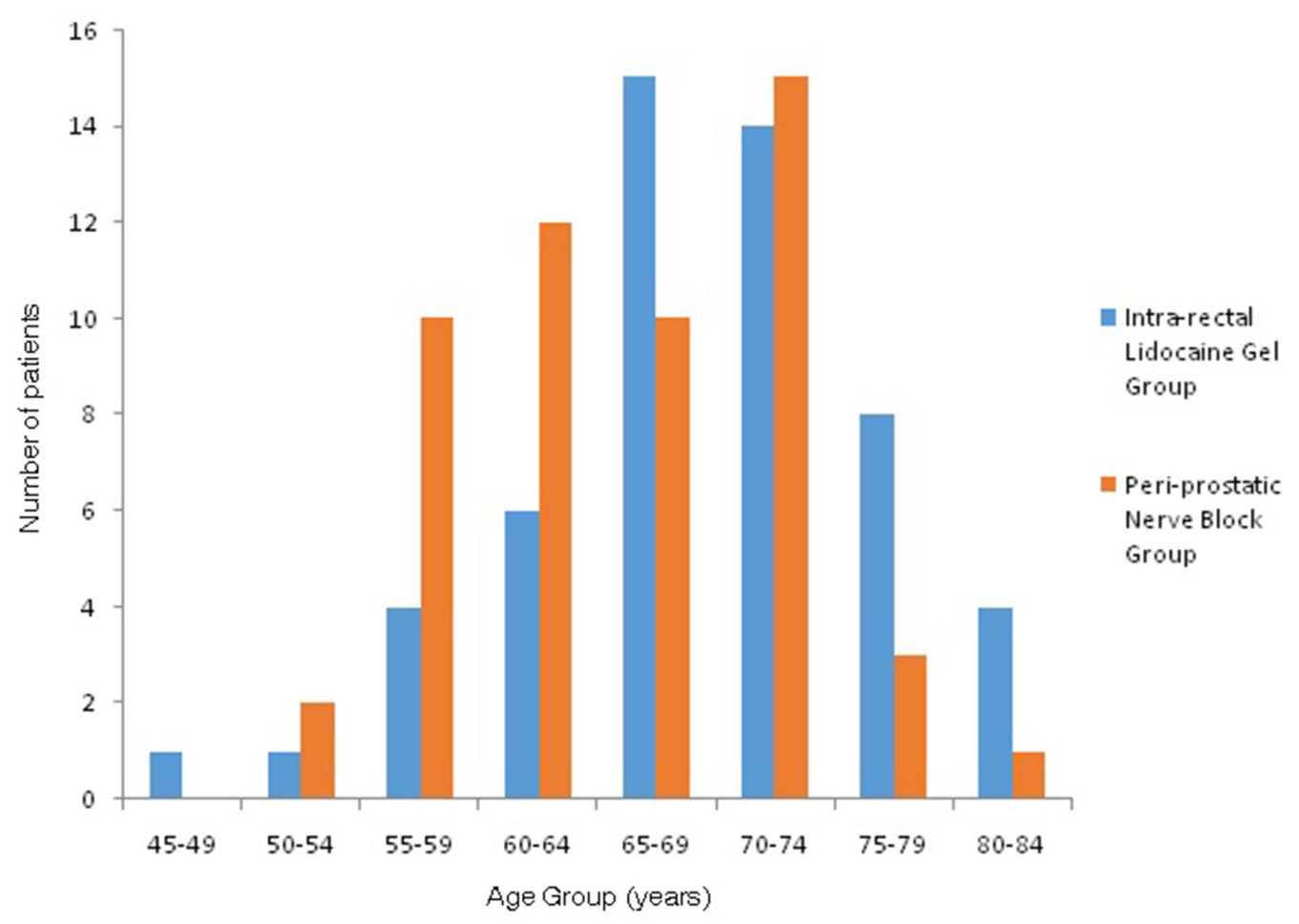

Fig. 1 Age group of patients who had prostate biopsy in the two study groups 
Table 1 Comparison of baseline characteristics of patients with suspected prostate cancer

\begin{tabular}{|c|c|c|c|}
\hline & Intra-rectal lidocaine gel group & Peri-prostatic nerve block group & $p$-value \\
\hline Age (years), mean $\pm S D$ & $68.1 \pm 7.8$ & $65.4 \pm 6.7$ & $0.055^{*}$ \\
\hline PSA (ng/ml), median [range] & $18.6[4.6-102.1]$ & $19.0[4.0-103.2]$ & $0.740^{\ddagger}$ \\
\hline Prostate volume (g), median [range] & $52.3[12.1-280.7]$ & $59.0[13.4-163.8]$ & $0.892^{\ddagger}$ \\
\hline
\end{tabular}

* $p$-value (independent samples $t$ test) $<0.05$ is significant

${ }^{*} p$-value (Mann-Whitney $U$ test) $<0.05$ is significant

SD standard deviation

\begin{tabular}{|c|c|c|c|}
\hline Outcome measures & $\begin{array}{l}\text { Intra-rectal } \\
\text { lidocaine gel } \\
\text { group }\end{array}$ & $\begin{array}{l}\text { Peri-prostatic } \\
\text { nerve block } \\
\text { group }\end{array}$ & $p$-value \\
\hline Pain during anaesthesia & $1.6 \pm 1.9$ & $3.7 \pm 2.1$ & $0.001^{*}$ \\
\hline $\begin{array}{l}\text { Pain during prostate } \\
\text { biopsy }\end{array}$ & $6.8 \pm 2.2$ & $2.9 \pm 1.9$ & $0.001^{*}$ \\
\hline $\begin{array}{l}\text { Satisfaction with pro- } \\
\text { cedure }\end{array}$ & $24(45.3 \%)$ & $46(86.8 \%)$ & $0.001^{\dagger}$ \\
\hline $\begin{array}{l}\text { Acceptance of a repeat } \\
\text { biopsy }\end{array}$ & $32(60.4 \%)$ & $47(88.7 \%)$ & $0.002^{+}$ \\
\hline
\end{tabular}

Pain score during anaesthesia administration and prostate biopsy are presented as mean \pm standard deviation, while other outcome measures are presented as frequency (\%)

* $p$-value (independent samples $t$ test) $<0.05$ is significant

${ }^{\dagger} p$-value (Chi-square test) $<0.05$ is significant
The most common post-biopsy complication was rectal bleeding (48.1\%). Other complications are displayed in Table 3.

\section{Discussion}

The definitive diagnosis of prostate cancer requires a prostate biopsy to obtain tissue for histopathologic confirmation. Prostate biopsy is an invasive procedure and is thus associated with pain. Different techniques of anaesthesia for prostate biopsy have been described and applied. However, opinions still vary regarding the optimal method of pain control. Local anaesthesia appears to be a simple, inexpensive and safe method of anaesthesia for prostate biopsy, and it was adopted in this study.

The demographic variables of the two groups in this study were essentially similar; the mean age for the intra-rectal lidocaine gel and the peri-prostatic nerve

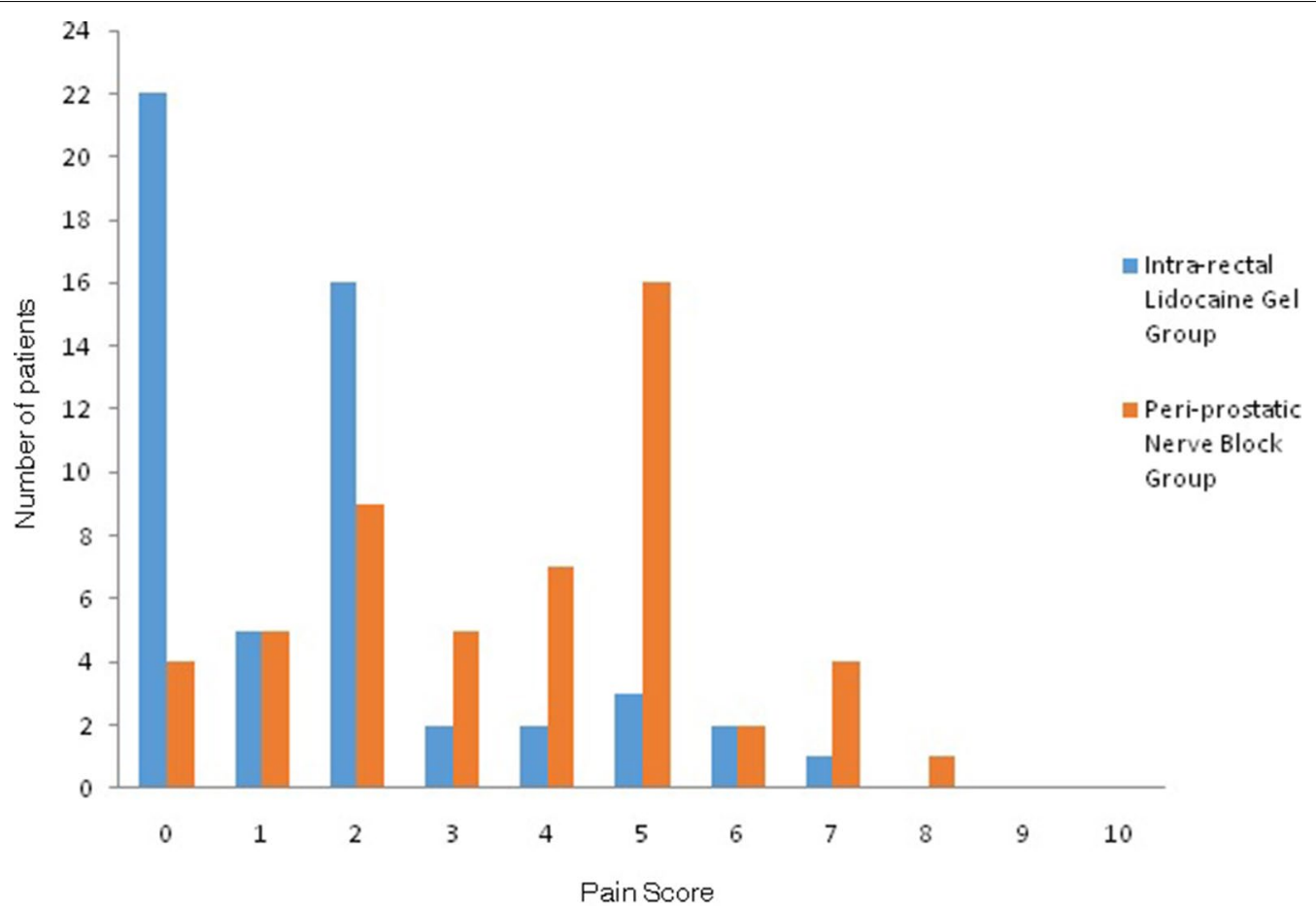

Fig. 2 Pain score during anaesthetic administration in the intra-rectal lidocaine gel and peri-prostatic nerve block group 


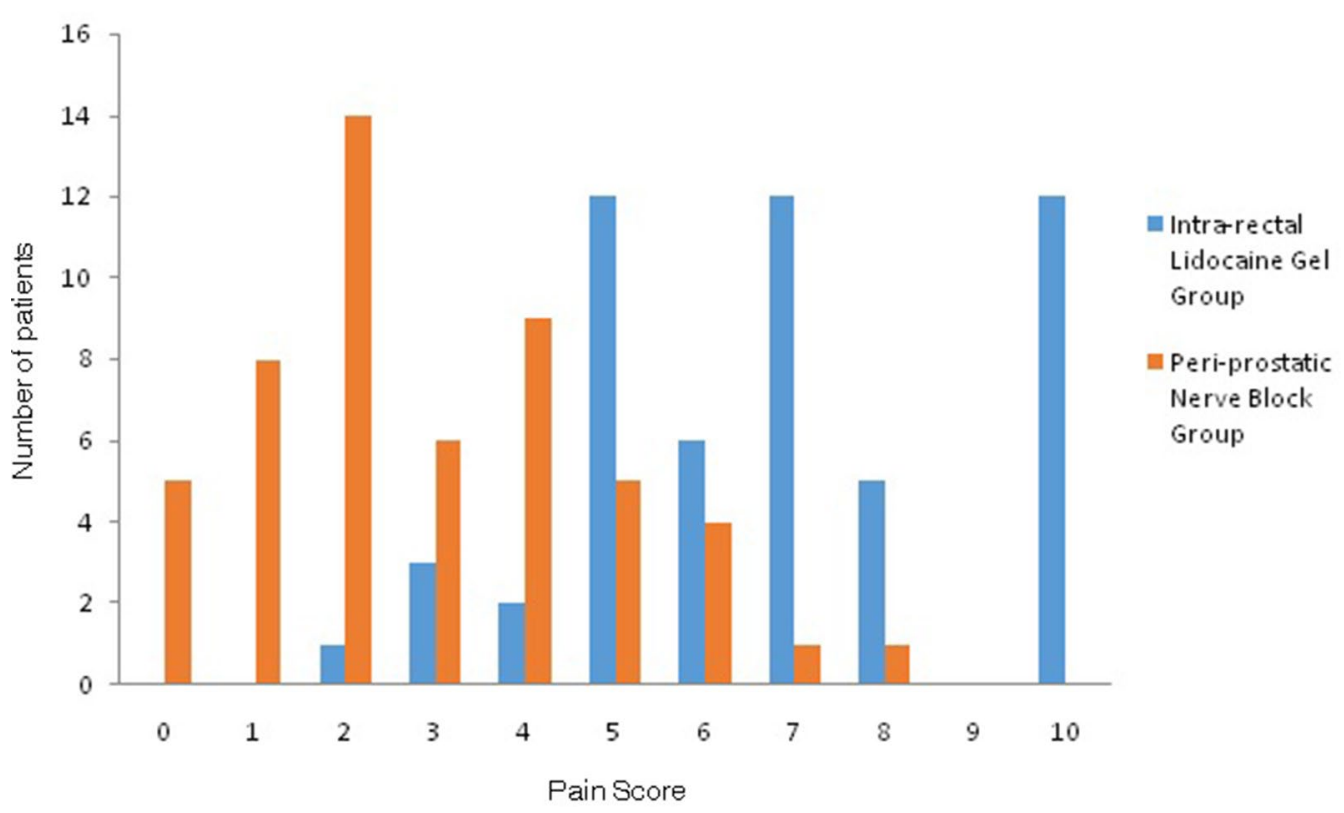

Fig. 3 Pain score during prostate biopsy in the intra-rectal lidocaine gel and peri-prostatic nerve block group

Table 3 Complications following prostate biopsy

\begin{tabular}{lll}
\hline Complications & Frequency & Percentage \\
\hline Rectal bleeding & 51 & 48.1 \\
Dysuria $^{\text {a }}$ & 34 & 41.5 \\
Haematuria $_{\text {Cysto-urethritis }}$ & 42 & 39.6 \\
Fever & 36 & 34.0 \\
Chills and rigours & 24 & 22.6 \\
Septic shock & 5 & 4.7 \\
Acute urinary retention $^{\text {b }}$ & 1 & 0.9 \\
\hline
\end{tabular}

a 41 patients in each group without urethral catheter were included in this proportion

b Total number of patients without catheter $=79$

block groups was 68.1 years and 65.4 years, respectively. This finding is similar to the mean age in other studies that compared the two local anaesthetic techniques [13, 17]. The median prostate-specific antigen and prostate volume were $18.6 \mathrm{ng} / \mathrm{ml}$ and $55.2 \mathrm{~g}$, respectively, and were also not significantly different between both groups. These values were higher than those reported in the study by Rodriguez et al., though large average prostate volume was reported by Hetta et al. in a North African population [13, 18].

This study is unique in the sense that it is one of the few studies that evaluated the tolerability of anaesthetic administration. Understandably, the mean pain score during anaesthetic administration was significantly lower in the intra-rectal lidocaine gel group than that in the peri-prostatic nerve block group (1.6 versus 3.7). This is because pain inevitably accompanies tissue infiltration of local anaesthetic compared to topical instillation of intra-rectal anaesthetic gel, in which other than the mild discomfort associated with dilatation of the anal verge at the insertion of the nozzle, it induces relatively little or no pain. However, Turgut et al. reported that about one-third of patients had pain during peri-prostatic nerve block with $72.9 \%$ of them having pain scores of less than 4 and no patient having pain score above 6 [19]. Unlike this study, basal periprostatic nerve block was used and it was not compared with another anaesthetic technique. In this study, a high pain score above 6 was recorded in 5 (9.4\%) of the patients in the peri-prostatic nerve block group. This could be related to the inadvertent stimulation of the rich sensory nerve fibres in the anal mucosa during needle introduction for the apical peri-prostatic nerve block [20, 21].

When we looked at the anaesthetic effect of intra-rectal lidocaine gel, it was sub-optimal while peri-prostatic nerve block provided superior pain relief during prostate biopsy with mean pain scores of 6.8 versus 2.9 , respectively. Hetta et al. recorded a mean pain score of 6.4 versus 3.0 in the intra-rectal lidocaine gel and peri-prostatic nerve block groups, respectively, which is in agreement with this study [18]. However, Trucchi et al. in their study to determine whether local anaesthesia reduces pain in trans-rectal prostate biopsy reported a much lower mean 
pain score of 5.5 and 0.5 in the intra-rectal lidocaine gel and peri-prostatic nerve block arm of the study, respectively [22]. In contrast to this study, they used carbocaine as the local anaesthetic agent. Carbocaine causes less vasodilatation and less absorption and has a longer duration of action and may thus be more effective than the lidocaine used in our study. Demir et al. reported a higher mean pain score of 8.2 in the intra-rectal lidocaine gel arm of their study which compared three different anaesthetic techniques for prostate biopsy [23]. However, the patients were not randomized into groups in their study.

Further evidence in support of the superiority of periprostatic nerve block in comparison to intra-rectal lidocaine gel, is the study of Rodriguez et al. and Alavi et al. that compared the efficacy of both local anaesthetic techniques $[13,17]$. The binding of lidocaine gel to the rectal mucosa might be responsible for its lower efficacy for prostate biopsy. On the other hand, the direct infiltration of the peri-prostatic nerves may have facilitated the superior pain relief provided by the technique.

The superiority of peri-prostatic nerve block over intrarectal lidocaine gel is also supported by the observation that $45.3 \%$ of patients who had intra-rectal lidocaine gel were satisfied compared to the statistically significant higher proportion of patients, $86.8 \%$, that were satisfied with a peri-prostatic nerve block. Naidoo et al. while comparing both methods of anaesthesia reported that $7.8 \%$ versus $2.4 \%$ of the patients had poor tolerance of prostate biopsy in the intra-rectal lidocaine gel and the peri-prostatic nerve block groups, respectively [20]. However, patients' tolerance of the procedure was assessed by six different operators unlike in this study where individual patients reported their satisfaction with the biopsy procedure.

Findings in this study which showed a significantly lower proportion of patients willing to accept a repeat biopsy if necessary in the intra-rectal lidocaine gel group $(60.4 \%)$ compared to the proportion in the peri-prostatic nerve block group (88.7\%) are similar to the observation of Tobias-Machado et al. who reported an acceptance rate for repeat biopsy of $60.0 \%$ for the intra-rectal lidocaine gel group [10]. However, a higher rate of acceptance of repeat biopsy (98.3\%) was recorded in the peri-prostatic nerve block arm of their study in comparison to the findings in this study. In contrast to this study, an acceptance rate of $87.0 \%$ versus $95.7 \%$ in the intra-rectal lidocaine gel and peri-prostatic nerve block groups has been reported by Naidoo et al. which was not significantly different between both groups [20]. However, the reported mean pain scores in that study, 3.1 versus 2.0 in the intra-rectal lidocaine gel and peri-prostatic nerve block groups, respectively, were much lower than those reported in this study. The significantly higher satisfaction rate and acceptance of repeat biopsy in the peri-prostatic nerve block group further reinforce the objective benefit of achieving a significantly lower pain score in this group.

Bleeding, infections and voiding complications were recorded following prostate biopsy in the present study. Although the rate of rectal bleeding reported in this study (48.1\%) was close to that reported by Ugwumba et al. (40.2\%), the rate of occurrence of fever was higher in the present study (22.6\% versus $8.9 \%$ ) [24]. This could be because their patients had antibiotic administration for 3 days after the biopsy which may have resulted in the lower rate of infection. The rate of haematuria (39.6\%) and acute urinary retention (1.3\%) were, however, within the range reported by Loeb et al. in a systematic review of prostate biopsy complications [25].

The limitation of this study was the fact that pain assessment was done at the end of the biopsy procedure rather than for each core of tissue taken during the procedure. It was also not feasible to blind the clinician performing the procedure to the technique of anaesthesia used. However, this would not affect the outcome of this study as the procedures were carried out in line with the study protocol and assessment of outcome measures was done by a blinded research assistant using objective measures.

\section{Conclusions}

Peri-prostatic nerve block is more effective and provides better patient satisfaction than intra-rectal lidocaine gel when used as anaesthesia for prostate biopsy. However, the administration of intra-rectal lidocaine gel induced little or no discomfort and was better tolerated by patients. Despite this moderate advantage, most patients still prefer the use of peri-prostatic block if they were to have a second biopsy.

\section{Acknowledgements}

Not applicable.

\section{Authors' contributions}

All authors were involved in the development of the project. MAT, LF, AS, $\mathrm{BKH}$ and $\mathrm{ON}$ were involved in data management. MAT and ATL did the data analysis and manuscript writing. MAT, MAH, MAW, AB and HYM carried out manuscript editing. All authors read and approved the final manuscript.

\section{Funding}

This study received no external funding.

\section{Availability of data and materials}

The datasets used and/or analysed during the current study are available from the corresponding author on reasonable request.

\section{Ethics approval and consent to participate}

Our manuscript is an excerpt from the first author's dissertation for which ethical approval was obtained from the Health Research Ethics Committee of Ahmadu Bello University Teaching Hospital, Shika-Zaria, Nigeria (ABUTH/ 
HREC/N17/2015), and written informed consent was obtained. Participation was voluntary, and confidentiality was maintained throughout the study.

\section{Consent for publication}

Not applicable.

\section{Competing interests}

The authors declare that they have no competing interests.

Received: 6 January 2020 Accepted: 2 June 2020

Published online: 28 August 2020

\section{References}

1. Bray F, Ferlay J, Soerjomataram I, Siegel RL, Torre LA, Jemal A (2018) Global cancer statistics 2018: GLOBOCAN estimates of incidence and mortality worldwide for 36 cancers in 185 countries. CA Cancer J Clin 0:1-31

2. Leung SYL, Wong BBW, Cheung MC, Ho KL, Lee FCW, Tam PC (2006) Intrarectal administration of lidocaine gel versus plain lubricant gel for pain control during transrectal ultrasound-guided extensive 10-core prostate biopsy in Hong Kong Chinese population: prospective doubleblind randomised controlled trial. Hong Kong Med J 12:103-107

3. Nazir B (2014) Pain during transrectal ultrasound-guided prostate biopsy and the role of periprostatic nerve block: what radiologists should know. Kor J Radiol 15:543-553

4. Akdere H, Burgazli KM, AktozT, Acikgoz A, Mericliler M, Gozen AS (2013) The importance of anatomical region of local anesthesia for prostate biopsy: a randomized clinical trial. Eur Rev Med Pharmacol Sci 17:2890-2895

5. Barbosa RAG, Da Silva CD, Torniziello MYT, Cerri LMDO, Carmona MJC, Malbouisson LMS (2010) A comparative study among three techniques of general anesthesia for ultrasound-guided transrectal prostate biopsy. Rev Bras Anestesiol 60:457-465

6. Antunes AA, Calado AA, Lima MC, Falcão E (2004) Efficacy of intrarectal lidocaine hydrochloride gel for pain control in patients undergoing transrectal prostate biopsy. Int Braz J Urol 30:380-383

7. Glass A, Punnen S, Shinohara K (2010) Local anesthesia for the prostate gland. In: Saadatniaki A (ed) Clinical use of local anesthetics. InTech Open Access, Rijeka, pp 59-74

8. Berger AP, Frauscher F, Halpern EJ, Spranger R, Steiner $H$, Bartsch $G$ et al (2003) Periprostatic administration of local anesthesia during transrectal ultrasound-guided biopsy of the prostate: a randomized, double-blind, placebo-controlled study. Urology 61:585-588

9. Griwan MS, Kumar A, Sen J, Singh SK (2012) Comparative evaluation of periprostatic nerve block and diclofenac patch in transrectal ultrasoundguided prostatic needle biopsy. Nephrourol Mon 4:560-564

10. Tobias-Machado M, Verotti MJ, Aragao AJ, Rodrigues AO, Borrelli M, Wroclawski ER (2006) Prospective randomized controlled trial comparing three different ways of anesthesia in transrectal ultrasound-guided prostate biopsy. Int Braz J Urol 32:172-180

11. García AM, Escudero JJ, Ramos de Campos M, Deltoro MF, Torrijos FS, Barrera AA et al (2009) Prospective randomized study on the use of lidocaine local anesthesia in prostate biopsy. Arch Esp Urol 62:339-347
12. Izol V, Soyupak B, Seydaoglu G, Aridogan IA, Tansug Z (2012) Three different techniques for administering analgesia during transrectal ultrasoundguided prostate biopsy: a comparative study. Int Braz J Urol. 38:122-128

13. Rodriguez A, Kyriakou G, Leray E, Lobel B, Guillé F (2003) Prospective study comparing two methods of anaesthesia for prostate biopsies: apex periprostatic nerve block versus intrarectal lidocaine gel: review of the literature. Eur Urol 44:195-200

14. Sahay SC, Gupta N, Singh P (2011) Comparison of pelvic plexus blockade to other conventional techniques of analgesia in transrectal ultrasound guided prostate biopsy. Afr J Urol 17:48-55

15. De Sio M, D'armiento M, Di Lorenzo G, Damiano R, Perdonà S, De Placido $S$ et al (2005) The need to reduce patient discomfort during transrectal ultrasonography-guided prostate biopsy: what do we know? BJU Int 96:977-983

16. Hawker GA, Mian S, Kendzerska T, French M (2011) Measures of adult pain. Arthrit Care Res 63:240-252

17. Alavi AS, Soloway MS, Vaidya A, Lynne CM, Gheiler EL (2001) Local anesthesia for ultrasound guided prostate biopsy: a prospective randomized trial comparing 2 methods. J Urol 166:1343-1345

18. Hetta WM, Niazi G, Elfawy D (2014) Local anesthesia by periprostatic block in transrectal ultrasound guided prostatic biopsy. Egypt J Radiol Nucl Med 45:137-142

19. Turgut AT, Olcucuoglu E, Kosar P, Geyik PO, Kosar U (2008) Complications and limitations related to periprostatic local anesthesia before TRUSguided prostate biopsy. J Clin Ultrasound 36:67-71

20. Naidoo A, Heyns CF, Aziz NA, Theron PD, Botha AA (2006) A prospective randomized study of periprostatic lignocaine injection versus intrarectal lignocaine gel or placebo gel for pain relief during transrectal ultrasound guided needle biopsy of the prostate. Afr J Urol 12:65-74

21. Maccagnano C, Scattoni V, Roscigno M, Raber M, Angiolilli D, Montorsi F et al (2011) Anaesthesia in transrectal prostate biopsy: which is the most effective technique? Urol Int 87:1-13

22. Trucchi A, De Nunzio C, Mariani S, Palleschi G, Miano L, Tubaro A (2005) Local anesthesia reduces pain associated with transrectal prostatic biopsy: a prospective randomized study. Urol Int 74:209-213

23. Demir A, Çeçen K, Karadağ MA, Uslu M, Arslan ÖE, Tarcan T (2015) Pain control and its relationship with histopathological outcome in TRUSguided prostate needle biopsy: a prospective non-randomized trial. J Urol Surg 2:86-90

24. Ugwumba F, Nnabugwu I, Echetabu K, Okoh A, Udeh E (2017) Rates and determinants of complications following trans-rectal prostate biopsy in Enugu, Nigeria. J Adv Med Res. 23:1-8

25. Loeb S, Vellekoop A, Ahmed HU, Catto J, Emberton M, Nam R et al (2013) Systematic review of complications of prostate biopsy. Eur Urol 64:876-892

\section{Publisher's Note}

Springer Nature remains neutral with regard to jurisdictional claims in published maps and institutional affiliations.

\section{Submit your manuscript to a SpringerOpen ${ }^{\odot}$ journal and benefit from:}

- Convenient online submission

- Rigorous peer review

- Open access: articles freely available online

- High visibility within the field

- Retaining the copyright to your article

Submit your next manuscript at $\gg$ springeropen.com 